\title{
Prospectiva del nuevo mundo educativo
}

\section{Prospective of the new educational world}

DOI: $10.46932 / \mathrm{sfjdv2n5-006}$

Received in: August 1st, 2021

Accepted in: September 30th, 2021

Marcel Oswaldo Méndez-Mantuano

Magíster en Gestión Ambiental

Instituto Superior Tecnológico Juan Bautista Aguirre

Daule - Ecuador

Amanda Yolanda Lozada Valdez

Ingeniera en Sistemas Computacionales

Instituto Superior Técnico Rey David

Daule - Ecuador

Jorge Christian Plaza Quizhpi

Ingeniero en Sistemas Administrativos Computarizados Instituto Superior Tecnológico Juan Bautista Aguirre

Daule - Ecuador

Carlos Alberto Almeida Alvarado

Magíster en Derecho Procesal

Director de Asesoría Jurídica de la Armada del Ecuador

Guayaquil - Ecuador

E-mail: calmeida56@hotmail.com

Jennifer Lisenya Mantuano Flores

Maestro en Administración de la Educación

Unidad Educativa Galo Plaza Lasso

Daule - Ecuador

E-mail:manflorl@hotmail.com

Danny Rafael Plúas Rogel

Maestro en Administración de la Educación

Unidad Educativa Galo Plaza Lasso

Daule - Ecuador

E-mail: danny_pluas00@hotmail.com

\author{
Karla Vanessa Ochoa Ladines \\ Economista \\ Unidad Educativa Galo Plaza Lasso \\ Daule - Ecuador \\ E-mail: kava2020@hotmail.com
}




\title{
RESUMEN
}

Alrededor del mundo se perciben cambios acelerados en todos los ámbitos que forman la cotidianidad, quienes modulan las nuevas estructuras relacionales entre personas y de éstas con su entorno. Estos cambios germinan de la acelerada transformación tecnológica, de la masificación de los medios de producción y de la globalización económica y social. Sin embargo, el ecosistema educativo parece haber sufrido una parálisis evolutiva, o al menos sus cambios no son percibidos con la misma intensidad que los derivados de los aspectos antes mencionados. Por lo tanto, es necesario dar una lectura aproximada de las nuevas realidades que se despliegan en la educación contemporánea. La presente investigación trata de abordar las ideas principales que se desarrollan en el libro "La educación en su laberinto: análisis y propuestas para una salida", el mismo que esboza las concepciones del denominado "Nuevo Mundo Educativo". Estos postulados representan la propuesta para el desarrollo de un modelo educativo que sea adaptable a los diferentes paradigmas educativos, sin llegar a denominarse una nueva teoría educativa. Se analizó la disputa hegemónica de la educación actual, la pedagogía que representa un equilibrio entre las nuevas ignorancias y los nuevos aprendizajes, y el detalle de la reconstrucción de la biografía docente.

Palabras claves: modelos educativos, mundo educativo, paradigmas educativos, prospectiva, teorías educativas.

\begin{abstract}
Accelerated changes are perceived around the world in all the areas that make up daily life, which modulate the new relational structures between people and between people and their environment. These changes germinate from the accelerated technological transformation, from the massification of the means of production and from economic and social globalization. However, the educational ecosystem seems to have suffered an evolutionary paralysis, or at least its changes are not perceived with the same inten sity as those derived from the aspects. Therefore, it is necessary to give an approximate reading of the new realities that are unfolding in contemporary education. The present investigation tries to approach the main ideas that are developed in the book "Education in its labyrinth: analysis and proposals for a way out", the same one that outlines the conceptions of the so-called "New Educational World". These postulates represent the proposal for the development of an educational model that is adaptable to the different educational paradigms, without being called a new educational theory. The hegemonic dispute of current education, the pedagogy that represents a balance between new ignorance and new learning, and the detail of the reconstruction of the teacher biography were analyzed.
\end{abstract}

Keywords: educational models, educational world, educational paradigms, prospective, educational theories.

\section{INTRODUCCIÓN}

A pesar de los altibajos, existen progresos en el sistema educativo, donde se intenta alcanzar mayores niveles de equidad y calidad educativa, a través de la eficacia en aras de acabar con las nuevas formas de discriminación y exclusión de las personas. Con el transcurso del tiempo, estas metas son cada vez más difíciles de cumplir, ya que los cambios sociales, culturales y tecnológicos exigen a los ámbitos educativos nuevas formas de interrelación, no obstante, la educación (en algunos casos) parece empecinada en mantener el dogmatismo formativo de los sujetos. Esta limitada transformación, es el 
resultado de los restringidos esfuerzos y recursos que son destinados para incitar los cambios requeridos de manera rápida y profunda (Echeita \& Verdugo, 2004).

La educación tiene como rol central la prevención y mitigación de las futuras crisis y conflictos sociales, además fortalece la paz y la democracia entre las naciones; finalmente es un puente conciliador para el fortalecimiento de la cohesión social a través de los derechos humanos. En definitiva, la educación es un arma noble que fomenta la identidad de la ciudadanía mundial, la tolerancia mutua, el civismo y el desarrollo ecoproductivo. También es un "elemento primordial de marco mundial integrado de los objetivos de desarrollo sostenible [...] una educación básica de calidad sienta las bases necesarias para el aprendizaje a lo largo de toda la vida en un mundo complejo y en rápida mutación" (Organización de las Naciones Unidas para la Educación, la Ciencia y la Cultura, 2015).

En distintas etapas del desarrollo moderno de las sociedades, estas han realizado marcados esfuerzos para replantear el orbe educativo, con el objetivo de diseñar una enseñanza que responda a la calidad y a la equidad, de tal manera que se centre en el impulso de las potencialidades individuales de las personas y no en la lógica empresarial que demanda de mano de obra y no de individuos. Los actuales matices generan dudas e incertidumbres sobre el rumbo que debe alcanzar la educación, ya que las transformaciones que en antaño duraban hasta generaciones, en la actualidad se hacen de manera vertiginosa y sin fundamentos previos, lo que hoy parece novedosos posiblemente en semanas o meses será anticuado y obsoleto. Los nuevos mapas políticos, sociales, culturales, económicos se dibujan y desdibujan de manera constante, variable e inestable, donde la educación es el anclaje de la sociedad y el fiel reflejo de ella, por lo tanto, no puede permanecer inconmutable a dichos escenarios y está obligada a reformularse para dar respuestas a las nuevas tendencias e inquebrantables desafíos (Castillo, 2015).

"Las sociedades que no se preparan para el futuro, que no ejercen el poder de su imaginación y se contentan con administrar su presente, están condenadas a vivir más de lo mismo" (Prigogine, 1983). Bajo estos preceptos, las sociedades modernas deben crear fundamentos que permitan la proyección analítica de escenarios futuros e inciertos de sus sistemas, incluyendo a la educación, lo cual ayudará a orientar los procesos de desarrollo.

Por lo tanto, a nivel educativo es preciso establecer herramientas de "prospectiva", las cuales permitan anticipar los efectos de las acciones presentes, a través de la construcción de futuros posibles con la ayuda de metodologías analíticas constituidas por procesos colectivos.

La prospectiva es definida como el proceso sistemático, participativo y constructivo a largo plazo que ayuda a definir acciones en el presente para construir el mejor de los futuros, la misma que se encuentra asociada a los procesos de planificación para el desarrollo sostenible. Los estudios prospectivos no tienen como finalidad predecir los hechos que sucederán, sino generar información sintetizada del 
pasado, presente y futuro para definir alternativas convenientes a la gran gama de situaciones (Secretaría Técnica Planifica Ecuador, 2019).

La percepción del ascenso de un nuevo paradigma educativo permite cuestionar elementos que de manera habitual han escoltado a los procesos de enseñanza y aprendizaje. Por ejemplo, las herramientas tecnológicas facilitan la visualización de conceptos que hasta hace poco eran intangibles para los estudiantes, además los conocimientos básicos están disponibles a un solo clic de distancia y los materiales didácticos son de fácil acceso y creación, todo esto conlleva a que los estudiantes sean más selectivos en la información que desean procesar (Campión, Maeztu, \& Andía, 2017).

Los conceptos y perspectivas anteriores ayudan a delinear las bases conceptuales sobre las cuales se fundamenta el presente análisis. El objetivo de este es realizar una síntesis de los principales postulados del libro "La educación en su laberinto: análisis y propuestas para una salida", el cual aborda el diseño de un "Nuevo Mundo Educativo" y cuyos autores son Romero, Pedro; Altisen, Claudio; Romero, José \& Noro, Jorge (2017).

\section{METODOLOGÍA}

Para el desarrollo de la investigación se aplicó un enfoque cualitativo, ya que únicamente se realiza un análisis de los postulados principales de los capítulos del libro "La educación en su laberinto: análisis y propuestas para una salida", donde posteriormente se efectuaron diagramas conceptuales para la esquematización y priorización de las ideas. También se realizaron mesas de diálogos entre los autores para examinar estos esquemas y diagramas. Este tipo de estudios no son estadísticos, ya que tiene elementos subjetivos que dependen de los juicios e ideas de los investigadores, donde las afirmaciones tienen fundamentos lógicos y coherencia con otros estudios.

El diseño es documental, el mismo que se concibe de la fundamentación bibliográfica de varios autores, principalmente de Romero, Pedro; Altisen, Claudio; Romero, José \& Noro, Jorge. Aunque el libro fue realizado en el 2017, el mismo cobra relativa importancia para la educación postpandemia, ya que urgen nuevas premisas que ayuden a la reconstrucción de la dinámica alumno-profesor.

\section{DESARROLLO}

\subsection{DISPUTA HEGEMÓNICA}

Dentro del entramado capitalista convergen dos actores diferenciados, denominados como sociedad política y sociedad civil. El primero ostenta el poder para reprimir ideologías divergentes que atentan contra su posición dominante, mientras que en el segundo grupo (en donde se encuentra la escuela, la iglesia y la prensa) figura la dirección simbólica y cultural. Todo esto converge en el reclamo implícito 
de parte de los estados hacia el sistema educativo, ya que estos destinan recursos económicos y a su vez demandan de cambios, sin embargo, estos son lentos y en algunos casos inobservables, por lo cual, se tiende a cuestionar la eficacia que tiene la educación en la generación de la plusvalía dentro de la sociedad. Según la lógica capitalista del costo-beneficio, los resultados no son satisfactorios, pero resulta paradójico que el mismo pensamiento mercantilista indique que la escuela es insustituible, a pesar de las mociones des-escolarizantes.

Los representantes escolares tienen parte dentro de esta disputa, donde estos idealizan y añoran la escuela del pasado y se muestran críticos a las adaptaciones espaciotemporales que ocurren dentro de las aulas en el "presente". Al percibir la historia, esta indica los reclamos que se han suscitado sobre los agentes educativos, pero es el mismo tiempo el que hace olvidar sus defectos y multiplica sus virtudes. Desde los años 20 y 40 del siglo pasado, aparecen nuevas innovaciones pedagógicas en los movimientos denominados como la "Escuela Nueva" o "Escuela Activa", no obstante, su alcance ha sido limitado y no han calado con profundidad en el sistema educativo. Entre sus principales exponentes destacan: Montessori, Agazzi y las hermanas Cossettini.

Existen 3 ciclos hegemónicos que formaron los paradigmas educativos, los cuales son:

1. Ciclo moderno: empezó desde la conformación de los estados nacionales y la europeización de los estilos de vida, además confluye en el creciente abandono de los campos hacia los grandes centros urbanos, derivado del modelo productivo de la revolución industrial. La escuela buscaba la homogeneización como una necesidad política, un modelo social y una de las razones del sistema escolar. Donde la familia poseía sus roles establecidos, las personas solo eran obreros de las grandes empresas y el "Progreso" era la utopía que ofrecía el sistema a cambio de la lealtad absoluta. Por lo tanto, la escuela es concebida como una herramienta estatal para perpetuar e incluso legitimar las desigualdades, mediante la homogeneización de los conocimientos y de los individuos.

2. Ciclo neoliberal: a partir de las décadas de los 60 y 70 surge el pensamiento neoliberal, como consecuencia de las medidas económicas que se originaron a partir de la guerra fría. Cuyos máximos exponentes globales fueron Ronald Reagan (Estados Unidos) y Margaret Thatcher (Reino Unido), incluso el estadounidense llegó a acuñar la frase “las nueve palabras más terroríficas en lengua inglesa son: Soy del Estado y aquí estoy para ayudar", cuyo objetivo era limitar las funciones del estado en el espacio cotidiano (liberalización de la economía, libre comercio, reducción del tamaño del estado, etc.). En el ámbito educativo, este modelo sustituyó de manera rápida al idealismo liberal del paradigma fundacional. Dentro de este ciclo, la educación debe ser eficaz y eficiente, cuyos resultados son medidos en términos de productividad y donde el 
problema no radica en la falta de los recursos, sino en el incorrecto manejo de estos. En otras palabras, si el producto-alumno no es satisfactorio, se debe a las fallas en la cadena formativa. La mejora del sistema está dada por la demanda del mercado laboral, donde el estado es el responsable del servicio y la oferta es realizada por los organismos privados. El neoliberalismo se encuentra vigente y es el modelo hegemónico, ya que posee una gran plasticidad para adaptarse de manera rápida a los cambios suscitados. Otro factor para su éxito es que los estados se han retirado del rol educador y se han transformado en meros garantes de las condiciones de juego del sistema.

3. Ciclo pos-estructuralista: el término fue acuñado por varios pensadores de finales del siglo XX reunidos en el "Coloquio Internacional sobre Lenguajes Críticos y Ciencias del Hombre". El paradigma evoca un lenguaje que pasa de la reflexión a lo performativo, como dos elementos constitutivos y no distantes entre sí. Su origen proviene del quiebre con el paradigma moderno, y sus críticas al sistema educativo han generado importantes análisis dentro del mismo, no obstante, el impacto de las medidas ha sido lacónico. Para este paradigma la escuela es el resultado de la modernidad, la misma que obedece a elementos históricos y por ende a la política, por lo tanto, representa la institucional de los constructos sociales válidos de la época, donde el reino de la verdad es relativo.

Las críticas que se realizan al sistema ayudan a desmitificar al mismo, debido a que posee su anclaje en la estructura política, económica, social y cultural. Por lo tanto, el paradigma del Nuevo Mundo Educativo no trata de crear nuevas estructuras conceptuales o mejorar ciertas experiencias o resultados escolares, sino que se fundamenta en las ideas prospectivas de un nuevo mundo desde los aspectos presentes, también en que los conceptos poseen una memoria cíclica y que el salto re-evolutivo (conjugación de dos necesidades: evolución y revolución) debe tener elementos de una reflexión temporal que vinculen al pasado, presente y futuro.

\subsection{LAS NUEVAS IGNORANCIAS Y LOS NUEVOS APRENDIZAJES}

Para los humanos el aprendizaje es inherente a su propia existencia, donde el conocimiento lo ha acompañado desde el inicio de su caminar por el planeta. Las ciencias surgieron a medida que las personas empezaron a apropiarse de su "yo" interior y de los medios de su entorno, siendo la única especie que diseñó una externalidad "internalizable”.

Existieron cuatro acontecimientos trascendentales en términos de aprendizaje en la educación:

1. La independencia de lo sobrenatural, donde la ciencia reemplaza a las creencias míticas.

2. La ciencia es utilizada como técnica productiva, a través de la cual se domina y transforma la naturaleza. 
3. La difusión de la escritura, la cual permitió dotar las cosas y hechos de temporalidad y espacialidad.

4. La institucionalización de la escuela, que reproduce los saberes y la constituyó en performativa, ya que es capaz de convertir la teorización en hechos que transmutan a la realidad.

Sobre el alumno gira la pedagogía única y lo identifica como el único sujeto de aprendizaje, en una sola dimensión que es el aula. El concepto de alumno fue delineado por la pedagogía moderna, la misma que encauza el aprendizaje de los niños y adolescentes bajo el control de los estados.

El Nuevo Mundo Educativo propone dos ejes sobre los cuales se deben desarrollar las acciones escolares. El primer eje se basa en el formato del dispositivo, el cual instituye las herramientas públicas de las nuevas metodologías de aprendizaje en los estudiantes. El segundo eje se relaciona con la cultura sobre la cual se educa, rediseñando los conceptos que se encuentran dentro y fuera del aula, para incorporar subjetividades que de manera clásica no han sido consideradas.

El fenómeno de la urbanización impone una nueva forma de interrelación entre las personas, en función de vínculos que trascienden la territorialidad. En las afueras de las fronteras escolares existen códigos sociales que no son incorporados dentro de los aprendizajes, su riqueza radica en la alta mutabilidad que presenta y que toda esta información es un termómetro de la misma sociedad, sobre sus percepciones, sus gustos y disgustos. Conviene reconocer que al exterior de las paredes de las escuelas existen individuos que construyen y deconstruyen información sin los límites tácitos impuestos por la ortodoxia docente.

En una época llena de paradojas, donde la religión funciona sin Dios, la política le agota al pueblo y la economía es incapaz de economizar los recursos disponibles, pareciera que el sistema educativo se hastío del alumnado. Sin embargo, el Nuevo Mundo Educativo reconceptualiza a los estudiantes y los posiciona como agentes participativos en la construcción de los conocimientos, sabiendo que dentro de las escuelas no se aprende todo lo necesario para los ciudadanos contemporáneos e interiorizando que no existen alumnos ni profesores a tiempo completo. Cabe puntualizar que los mayores aprendizajes se logran durante los primeros años de escolaridad, a medida que transcurren los niveles, el aprendizaje se realiza de manera superficial. Nicolas Carr argumenta que este fenómeno nace con el zapping de la información que nos somete el internet.

El aprendizaje tradicional ponía barreras dialécticas entre la ciencia que enseñaba el distanciamiento con las personas y la naturaleza. El nuevo aprendizaje nos pone en cercanía con el mundo que nos rodea, a través del sentido fisiognómico que se basa en la intimidad del saber cómo una relación entre agentes activos y no con objetos inertes. Se han delimitado cuatro mutaciones del conocimiento 
desde los aspectos tradicionales a los contemporáneos: de lo sagrado a lo profano, de lo pesado a lo liviano, de racional a sensible y de los cosmológico a lo cotidiano.

La escolaridad clásica intentaba superar las ignorancias a través de la ciencia des-barbarizante, quien debía cumplir con los dogmas preestablecidos del sistema y basarse en los rigurosos protocolos. Por su parte el Nuevo Mundo Educativo identifica las "nuevas ignorancias", como aquellos conocimientos para la vida y su relación de cotidianidad con los sujetos.

A las nuevas ignorancias se las ha catalogado en ignorancias de los fenómenos, ignorancias de las cosas e ignorancia de los futuros, la primera hace referencia al impacto generado por la cultura y la tecnología que son difíciles de asimilar debido a su rápida ocurrencia. Mientras que el segundo término resalta la importancia de los dispositivos de aprendizaje que ayudan al entendimiento de la información. Finalmente, la ignorancia de los futuros menciona la importancia del direccionamiento generacional (a los Ni-Ni, ni estudian ni trabajan), para sacarlos del cansancio educativo y laboral.

\subsection{ENTRE LAS NUEVAS IGNORANCIAS Y LOS NUEVOS APRENDIZAJES}

El Nuevo Mundo Educativo propone una pedagogía que muta de manera constante, capaz de incorporar elementos del pasado y reformularse en el presente, entre las características que deben predominar se encuentra: universalidad, multiplicidad, heterogeneidad, polifuncionalidad, movilidad, permeabilidad, autodeterminación, autocontrol, capacidad para el servicio de los actores, flexibilidad en los tiempo, adaptabilidad a los sujetos que aprenden, adaptabilidad con las tecnologías emergentes, valorización de las características individuales, que permita el ocio, entre otras.

La pedagogía única donde el profesor tiene que preparar el material de trabajo, determinar el proceso de enseñanza y aprendizaje, realizar las actividades de evaluación y acreditación y efectuar la retroalimentación; debe sufrir un proceso de expansión que permita mayor inclusión de las subjetividades que quedan fuera de las aulas, ya que todas las áreas de análisis, planificación y desarrollo giran alrededor de la visión de un único actor, el profesor. Una pedagogía que se encierra en los sujetos suele ser elitista y prohibitiva, por ello, las pedagogías deben mostrarse expansivas con la capacidad de incluir una gran cantidad de hermenéuticos, incluso alguno de ellos fuera de las fronteras educativas. También debe retroalimentarse de las opiniones análogas y de las divergentes, que permitan su construcción y deconstrucción de manera permeable a través de su temporalidad y espacialidad.

El Nuevo Mundo Educativo propone cuatro tipos de pedagogías que permitan diferenciar el tipo del trabajo docente, el compromiso educativo, la organización institucional y el ordenamiento de los tiempos y los espacios, a través de las denominadas:

- Pedagogía del diseño, orientada a la creación de ambientes de aprendizajes. 
- Pedagogía de la comunicación, hace referencia al diseño de diálogos persuasivos entre docentes y estudiantes.

- Pedagogía del entretenimiento, diseña entornos que propician los aprendizajes a través de la diversión.

- Pedagogía de la exploración, ayudan a indagar formas de integración de las prácticas con su abordaje en los asuntos comunes.

Donde cada pedagogía detalla los momentos de la función docente, a diferencia de la pedagogía única que concentraba sus funciones, generando improductividad por la multiplicidad de las tareas.

Las cuatro pedagogías no son una fragmentación de la pedagogía tradicional, sino una construcción dialéctica y conceptual de cada una de las estas, donde existen particularidades que deben desarrollarse para poder ejecutarse de manera coherente, además libera al docente de la realización individual de todas las funciones, siendo parte de un engranaje que permite y recrea el movimiento de una determinada pedagogía, a través de la responsabilidad compartida.

Al ser la evaluación uno de los ejes más cuestionados, el paradigma del Nuevo Mundo Educativo propone un mecanismo denominado como Distritos Integrales de Aprendizajes Sociales (DIAS), el cual registrará la evaluación de forma permanente y de forma diversificada en base a los diferentes aprendizajes que se desarrollen dentro del aula.

\subsection{RECONSTRUCCIÓN DE LA BIOGRAFÍA DOCENTE}

Existen muchas discusiones sobre las funciones y capacidades que debe poseer un docente, sin embargo, bajo el modelo capitalista y en nombre de la calidad, el profesorado debe ser "productivo", donde se utilizan mecanismos burocráticos para su control y se evalúa de manera acechante su productividad, como mero instrumento de la reproducción de conocimientos aprobados por el sistema, además de socavar todo intento de ilegitimar los procesos que se llevan a cabo dentro y fuera de la escuela.

Para el sistema tradicional, la docencia está orientada a la capacitación y al ejercicio de los conocimientos instrumentales, los cuales deben ser útiles dentro del mercado laboral, por ello, se desvaloriza la educación de tipo humanística para evitar los cuestionamientos del sentido de muchas acciones y así empoderar a la gestión por encima de la razón. El discurso redundante insiste que los docentes deben ser personajes solitarios, fieles cumplidores de sus roles determinados por los reglamentos, dirigentes eficientes de los saberes, debe estar alineado a las estabilidades, predecible, dócil, obediente, disciplinado, motivador y cuyo modelo de trabajo se encuentre dentro del modelo para la producción industrial. 
Todo lo anterior provoca que los docentes muestran mayores distancias en la adquisición de nuevos conocimientos, donde sus esfuerzos deben limitarse a encontrar "recursos didácticos" que sean atractivos o novedosos sobre conocimientos inmóviles. Las presiones provenientes del interior y del exterior de la escuela, genera que más de la mitad de los docentes quiera cambiar sus trabajos, los mismos que muestran altos niveles de insatisfacción por haber escogido la carrera educativa, además dos tercios no consideran necesario realizar capacitaciones de actualización (encuesta realiza en la ciudad de RosarioArgentina a docentes de niveles primarios y secundarios). Poco a poco el docente se asocia con la idea que sus acciones no calan en el sistema, de tal manera que son nómadas exiliados que recorren territorios colonizados por las lógicas institucionales totalizadoras y enajenantes, a las cuales no les interesa las particularidades de las singularidades en las personas. Quizás muchos se identifican con las palabras de Byung Chul Han, quien menciona:

"La proliferación de lo igual es lo que constituye las alteraciones patológicas de las que está
aquejado el cuerpo social... La expulsión de lo distinto pone en marcha un proceso destructivo
totalmente diferente: la autodestrucción. En general impera la dialéctica de la violencia: un
sistema que rechaza la negatividad de lo distinto desarrolla rasgos autodestructivos" (Han, 2017).

A pesar de las críticas realizadas al mundo educativo, el docente es un personaje relativamente valorado y nadie duda en la importancia que tiene en la construcción de las sociedades, por lo tanto, nadie se atreve a eliminar su figura dentro del andamiaje colectivo.

Tal como existen políticas integradoras de los estudiantes, el Nuevo Mundo Educativo propone políticas institucionales de inclusión docente, las cuales estarán enmarcadas en la creación de estímulos que fomenten la pertinencia, el acompañamiento y el sostenimiento de los maestros dentro del sistema. Se debe trabajar en la biografía docente, que permita a los sujetos reconocerse a través de las múltiples transformaciones que sufrirá dentro de toda su vida laboral, lo que propiciará un involucramiento activo en la creación de los procesos educativos. La carrera del docente es otro de los aspectos que deben ser reformulados, de tal manera, que el docente no sea un sujeto sedentario sino nómada, es decir, que tenga la posibilidad de realizar actividades diversas dentro de un marco institucional ajustable y no inflexible, que piense en los sujetos no como objetos ni como instrumentos, sino como personas con la capacidad de diseñar sociedades empáticas que busquen el bien común. La formación docente debe fundamentarse en una dinámica cotidiana que permita la operacionalización de las ideas y la adquisición de nuevas competencias que nazcan desde la iniciativa personal y no percibidas como una obligatoriedad de las instituciones. Al hablarse de inclusión, esto demanda de condiciones óptimas que permitan la simbiosis entre las orientaciones sistemáticas, sin tratar de ahogar o homogeneizar la creatividad y las particularidades individuales de los sujetos. 


\section{CONCLUSIONES}

Dentro de la educación existen varios paradigmas educativos, los cuales identifican las metodologías aplicadas y las experiencias educativas logradas, dentro de un marco histórico y social. Estas ayudan a interiorizar las causas y efectos que poseen estos procedimientos en los estudiantes. Por su parte, la prospectiva es un ejercicio metodológico y analítico que permite determinar las acciones futuras, tomando como referencia el pasado y el presente.

El libro "La educación en su laberinto: análisis y propuestas para una salida", analiza de manera congruente los diferentes momentos históricos sobre los cuales se viene desarrollando la actividad educativa, y con ello realiza una proyección de un modelo formativo denominado "Nuevo Mundo Educativo". La importancia de esta propuesta es que no posee rasgos utópicos, sino que propone un trabajo desde el interior del actual sistema, a través del conocimiento de la existencia de la hegemonía capitalista sobre los ámbitos formativos, del desarrollo de nuevos aprendizajes que respondan a las nuevas preguntas que se desarrollan desde la contemporaneidad, y de la reconstrucción de la imagen clásica del docente como un agente dinámico con particularidades individuales.

A manera de síntesis, el libro esquematiza una "obsolescencia educativa cíclica", de tal manera, que ciertos rasgos metodológicos pueden ser reutilizados, pero sin ser idealizarlos, ya que estos sufren constantes cambios derivados de la hiperconectividad antrópica del mundo actual. En otras palabras, se pueden tomar elementos educativos del pasado, reestructurarse en el presente y proponerlos en acciones futuras.

El Nuevo Mundo Educativo es un modelo "líquido", el cual se ajusta a las particularidades y es flexible en su sistematización, sin embargo, existen elementos "sólidos" como las pedagogías del diseño, de la comunicación, del entretenimiento y la exploración, las mismas que forma su piedra angular metodológica. 


\section{REFERENCIAS}

Campión, R., Maeztu, V., \& Andía, L. (2017). Los contenidos digitales en los centros educativos: Situación actual y prospectiva. Revista Latinoamericana de Tecnología Educativa, 16(1), 51-66. doi:10.17398/1695-288X.16.1.51

Castillo, C. (2015). La educación inclusiva y lineamientos prospectivos de la formación docente: una visión de futuro. Revista Actualidades Investigativas en Educación, 15(2), 1-33. doi:10.15517/AIE.V15I2.18534

Echeita, G., \& Verdugo, M. (2004). La Declaración de Salamanca sobre Necesidades Educativas Especiales 10 años después. Salamanca: Inico. Obtenido de http://riberdis.cedd.net/bitstream/handle/11181/3223/Declaracion_de_Salamanca.pdf?sequence=1\&rd=0 031847251956687

Han, B. C. (2017). La expulsión de lo distinto. Barcelona: Herder.

Organización de las Naciones Unidas para la Educación, la Ciencia y la Cultura. (2015). Enseñanza y Aprendizaje: Lograr la Calidad para todos. Obtenido de https://unesdoc.unesco.org/ark:/48223/pf0000225654_spa

Romero, P., Altisen, C., Romero, J., \& Noro, J. (2017). La educación en su laberinto: análisis y propuestas para una salida. Rosario: Ediciones HomoSapiens.

Secretaría Técnica Planifica Ecuador. (2019). Propuesta metodológica para la planificación prospectiva territorial de los gobiernos autónomos descentralizados. Quito: Secretaría Técnica Planifica Ecuador. Obtenido de https://www.planificacion.gob.ec/wp-content/uploads/downloads/2019/09/Caja-deherramientas-Prospectiva-Final.pdf 